\title{
Mercury analysis in hair: Comparability and quality assessment within the transnational COPHES/DEMOCOPHES project
}

\author{
Marta Esteban, Birgit K. Schindler, José A. Jiménez-Guerrero, Holger M. Koch, \\ Jürgen Angerer, Teresa C. Rivas, Montserrat Rosado, Silvia Gómez, Ludwine Casteleyn, \\ Marike Kolossa-Gehring, Kerstin Becker, Louis Bloemen, Greet Schoeters, Elly Den Hond, \\ Ovnair Sepai, Karen Exley, Milena Horvat, Lisbeth E. Knudsen, Anke Joas, Reinhard Joas, \\ Dominique Aerts, Pierre Biot, Daniela Borošová, Fred Davidson, Irina Dumitrascu, \\ Marc E. Fisher, Margaretha Grander, Beata Janasik, Kate Jones, Lucie Kašparová, \\ Thorjørn Larssen, Miklos Naray, Flemming Nielsen, Philipp Hohenblum, Rui Pinto, \\ Catherine Pirard, Gregory Plateel, Janja Tratnik, Jürgen Wittsiepe, Argelia Castaño*, \\ EQUAS Reference Laboratories ${ }^{1}$
}

\section{A R T I C L E I N F O}

\section{Article history:}

Received 20 May 2014

Received in revised form

21 October 2014

Accepted 14 November 2014

Keywords:

COPHES/DEMOCOPHES

Biomonitoring

Quality assurance

Hair

Mercury

Minamata convention

\begin{abstract}
A B S T R A C T
Human biomonitoring (HBM) is an effective tool for assessing actual exposure to chemicals that takes into account all routes of intake. Although hair analysis is considered to be an optimal biomarker for assessing mercury exposure, the lack of harmonization as regards sampling and analytical procedures has often limited the comparison of data at national and international level. The European-funded projects COPHES and DEMOCOPHES developed and tested a harmonized European approach to Human Biomonitoring in response to the European Environment and Health Action Plan.

Herein we describe the quality assurance program (QAP) for assessing mercury levels in hair samples from more than 1800 mother-child pairs recruited in 17 European countries. To ensure the comparability of the results, standard operating procedures (SOPs) for sampling and for mercury analysis were drafted and distributed to participating laboratories. Training sessions were organized for field workers and four external quality-assessment exercises (ICI/EQUAS), followed by the corresponding web conferences, were organized between March 2011 and February 2012. ICI/EQUAS used native hair samples at two mercury concentration ranges $(0.20-0.71$ and $0.80-1.63)$ per exercise. The results revealed relative standard deviations of $7.87-13.55 \%$ and $4.04-11.31 \%$ for the low and high mercury concentration ranges, respectively. A total of 16 out of 18 participating laboratories the QAP requirements and were allowed to analyze samples from the DEMOCOPHES pilot study. Web conferences after each ICI/EQUAS revealed this to be a new and effective tool for improving analytical performance and increasing capacity building. The
\end{abstract}

\footnotetext{
Abbreviations: AAS, Atomic Absorption Spectrometry; COPHES, Consortium to Perform Human Biomonitoring on a European Scale; CV-AAS, Cold Vapor Atomic Absorption Spectrometry; CV-AFS, Cold Vapor Atomic Fluorescence Spectrometry; DEMOCOPHES, Demonstration of a Study to Coordinate and Perform Human Biomonitoring on a

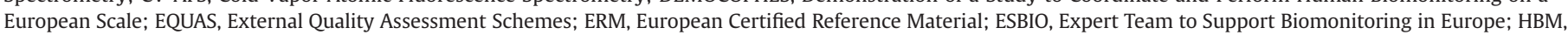

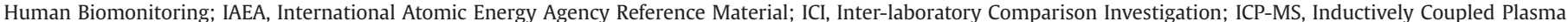

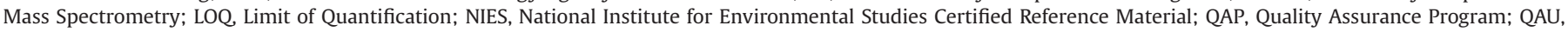
Quality Assurance Unit; RL, Reference Laboratory; RSD, Relative Standard Deviation; SOP, Standard Operating Procedures; TWI, Tolerable Weekly Intake

* Corresponding author. Fax: +34915097029.

E-mail address: castano@isciii.es (A. Castaño).

${ }^{1}$ Masatake Fujimura, National Institute for Minamata Disease (NIMD), Japan.

Alain LeBlanc, Centre de Toxicologie du Québec (CTQ) of the Institut national de santé publique du Québec (INSPQ), Canada.

Keith Levine, RTI International, USA.

Iouri Romachine, First Nations and Inuit Health Branch (FNIHB) Laboratory, Canada.

David Verbrugge, Alaska State Public Health Laboratory, Alaska.

Grazyna Zareba, University of Rochester, USA.
} 
procedure developed and tested in COPHES/DEMOCOPHES would be optimal for application on a global scale as regards implementation of the Minamata Convention on Mercury.

(c) 2014 Elsevier Inc. All rights reserved.

\section{Introduction}

Mercury is a well-known toxin and its presence in the environment and in the human food chain is a matter of increasing concern. In light of this, the Minamata Convention, a global action to protect human health and the environment by reducing exposure to mercury and mercury compounds, was signed in October 2013. Article 19 of this Treaty calls for harmonized methodologies for, amongst others, monitoring mercury levels in the population (http://www.mercuryconvention.org).

Mercury contamination is a worldwide problem because of its long-range transport and its ubiquity in global marine ecosystems, thus meaning that the entire global population is potentially exposed. Indeed, the general population, with non-occupational exposure, is mainly exposed to mercury via food, with fish being the major dietary contributor, and to a lesser extent by amalgam fillings (UNEP/WHO, 2002). According to data from the EFSA, high fish consumers may exceed the tolerable weekly intake (TWI) for methylmercury (MeHg) of $1.3 \mu \mathrm{g} \mathrm{Hg} / \mathrm{kg}$ b.w. by up to sixfold (EFSA, 2012).

The analysis of mercury levels in hair could become a highly recommended tool for monitoring mercury levels in the general population. Methylmercury is the main chemical form of mercury in fish and its analysis in hair is accepted as a reliable estimate of the internal dose (Harkins and Susten, 2003). Methylmercury is incorporated into the follicle during hair formation and shows a direct correlation with blood levels (Clarkson and Magos, 2006; UNEP/WHO, 2008). Moreover, as it does not return to the blood once incorporated, this matrix can give information about the history of exposure by analyzing different longitudinal sections of hair strands (UNEP/WHO, 2008). Hair is a non-invasive matrix that is easy to sample and does not require highly trained technicians. In addition, hair samples can be transported and conserved at room temperature, thereby facilitating logistics during study fieldwork. With regard to storage, the methylmercury content in hair in long-term storage remains unchanged over many years provided the sample is stored under dry and dark conditions at room temperature (Horvat et al., 2012). However, the fact that methylmercury analysis is time-consuming and expensive means that its use in large human biomonitoring (HBM) surveys is limited. The measurement of total mercury in hair is generally accepted as a surrogate for methylmercury exposure as it is present in hair in a high percentage (approx. $80 \%$ or more) and analysis at different concentrations is simpler and cheaper (Clarkson and Magos, 2006; McDowell et al., 2004; Berglund et al., 2005; UNEP/WHO, 2008; Poulin and Gibbs, 2008; EFSA, 2012). In addition, reference material for mercury in hair analysis is available (e.g. NIES, ERM or IAEA), an aspect that is essential when it comes to validating analytical methods.

Despite the multiples advantages of using hair in HBM surveys, there are some relevant influencing factors, such as hair treatments (coloring, curling, etc.), race (ethnicity) and, most importantly, external contamination if participants are living in contaminated areas (hot-spots) (ATSDR, 2001; McDowell et al., 2004; Dakeishi et al., 2005; UBA, 2005). However, overall, and irrespective of the human matrix used (hair, urine, blood, etc.), the effect of the different sampling and sample-preparation procedures and analytical methods used can influence the results significantly (ATSDR, 2001).
Mercury concentration in hair can be determined by different methods, with the preferred technique being Atomic Absorption Spectrometry (AAS) (UNEP/WHO, 2008). Recently, direct mercury analyzers are becoming more and more popular given the advantage of not requiring sample pretreatment or extraction and the short analysis times, thus allowing high sample throughput. The hair analysis panel discussion arranged by the Agency for Toxic Substances and Disease Registry in 2001 (ATSDR, 2001) encouraged the development of Standard Operating Procedures (SOPs) to standardize sampling and chemical analysis as well as the need to collect exposure histories, establish quality-assurance protocols and develop external validation by means of proficiency testing as this would lead to more reliable and reproducible results in hair analysis. Some years later, the German Federal Environmental Agency again pointed out the need to establish external quality control for hair analysis as well as SOPs (UBA, 2005).

In a wider framework, the European Environment and Health Action Plan 2004-2010 identified the high variability in HBM activities in Europe as a problem and called for "the development of a coherent approach to HBM in Europe" (EHAP, 2004). Activities aimed at harmonizing HBM in Europe commenced in 2007 with the establishment of Expert Team to Support Biomonitoring in Europe (ESBIO) (ESBIO, 2009), a precursor to the EU twin-projects Consortium to Perform Human Biomonitoring on a European Scale (COPHES) and Demonstration of a Study to Coordinate and Perform Human Biomonitoring on a European Scale (DEMOCOPHES). Thus, the theoretical basis required for such harmonization was developed in COPHES (Joas et al., 2012; COPHES website) and tested through the DEMOCOPHES pilot study (Becker et al., 2014; DEMOCOPHES website). A total of 17 countries participated in this test phase, in which mercury in hair and cadmium, cotinine, phthalate metabolites and, for some countries, bisphenol A in urine were analyzed in samples from mother-child pairs under a strict quality-assurance program.

The activities for harmonizing urinary parameters were presented and discussed in a previous paper by Schindler et al. (2014). The objective of this manuscript is to show the measures taken to ensure the reliability and comparability of hair mercury data among countries participating in DEMOCOPHES.

\section{Material and methods}

A Quality Assurance Unit (QAU) was established within the framework of COPHES/DEMOCOPHES by the leaders of the work package in charge of sample handling, analysis and biobanking (WP 3). The QAU comprised experts from the Instituto de Salud Carlos III (ISCIII, Madrid, Spain) and the Institute for Prevention and Occupational Medicine of the German Social Accident Insurance - Institute of the Ruhr - University Bochum (IPA, Bochum, Germany).

The QAU was responsible for all tasks related to quality control and quality assurance of the pre-analytical and analytical phases and served as a reference point for all technical questions that arose during the DEMOCOPHES pilot study (Schindler et al., 2014).

The 18 laboratories participating in the quality assurance program were from Austria, Belgium, the Czech Republic, Denmark, Germany, Hungary, Ireland, Luxembourg, Norway, Poland, Portugal, Romania, Slovakia, Slovenia, Spain, Sweden, Switzerland and the United Kingdom. 
A glossary of pre-analytical and analytical terms was delivered to all laboratories at the commencement of the project to ensure that participants used the same language.

\subsection{Harmonization measures in the pre-analytical phase}

\subsubsection{Pre-analytical standard operating procedures}

In order to harmonize sampling, the QAU drafted a SOP that included detailed instructions and specified different ways to proceed depending on the length of the hair. A video of the hair sampling procedure employed can be viewed on the web page of the CNSA (ISCIII) (http://www.eng.isciii.es/ISCIII/es/contenidos/fdel-instituto/fd-organizacion/fd-estructura-directiva/fd-subdirec cion-general-servicios-aplicados-formacion-investigacion/fd-cen tros-unidades/fd-centro-nacional-sanidad-ambiental/fd-servicioscientifico-tecnicos_sanidad-ambiental/s-c-t-cnsa-toxicologia-am biental.shtml).

Two additional pre-analytical SOPs, one defining the requirements for sample conservation and packing and the other focussed on sample reception and registration, were drafted and provided to the participants (see COPHES/DEMOCOPHES web page, http:// www.eu-hbm.info/cophes/project-work-packages/trainings-agen das-and-presentations).

\subsection{Harmonization measures in the analytical phase}

\subsubsection{Analytical SOP}

The QAU provided a SOP with clear, concise and comprehensive written instructions for direct mercury analysis in hair by thermal decomposition-gold amalgamation atomic absorption spectroscopy (http://www.eu-hbm.info/cophes/copy_of_Annex4.1SOPMercuryin hair.pdf). This SOP was validated in different inter-laboratory exercises and certified under ISO17025 standard by the Spanish National Accreditation Body (ENAC) (ISO/IEC 17025, accreditation number 223/ LE460). The method had a limit of quantification of $0.01 \mu \mathrm{g} \mathrm{Hg} / \mathrm{g}$ hair in samples of $100 \mathrm{mg}$. Participating laboratories used it as a basis for drafting their own SOP. Considering the short time for implementing the pilot study, participating laboratories were allowed to use their own analytical methods but, to ensure the comparability of the results, four external quality-assurance exercises were organized in less than a year.

\subsubsection{Control materials}

A wide range of concentrations was expected in the DEMOCOPHES target population as mercury levels can vary from $<0.5 \mu \mathrm{g} / \mathrm{g}$ for non-fish eaters to $1-2 \mu \mathrm{g} / \mathrm{g}$ for those with low to moderate consumption. As such, the control material employed in the external quality assessment exercises had mercury concentrations in the range $0.20-1.63 \mu \mathrm{g} / \mathrm{g}$. Control materials were prepared from native hair obtained from either a single sample or different hair samples with similar levels of mercury.

Samples were carefully cut into small pieces (1-3 mm) using titanium scissors and aliquoted into glass vials. Twenty of these vials were randomly selected in order to check homogeneity and then stored at room temperature under dry and dark conditions. They were subsequently analyzed to check stability for more than a month. All analyzes were performed by direct mercury analysis via thermal decomposition-gold amalgamation atomic absorption spectroscopy (DMA-80 Direct Mercury Analyzer, Milestone, USA) and following the analytical method described in the SOP delivered to the participating laboratories (http://www.eu-hbm.info/ cophes/copy_of_Annex4.1SOPMercuryinhair.pdf). Two different reference materials, namely NIES-13 and IAEA 086, with mercury concentrations of 0.573 and $4.42 \mu \mathrm{g} / \mathrm{g}$ respectively, were used to characterise the control material. Preparation and analysis of the control material was performed in the Environmental Toxicology
Unit of the National Center for Environmental Health (ISCIII, Spain), which developed the SOP and has successfully participated in regular international inter-laboratory comparison exercises since 2008 .

\subsubsection{External quality-assessment exercises}

Four external quality-assurance exercises were organized between March 2011 and February 2012: two Inter-laboratory Comparison Investigations (ICIs) and two External Quality-Assessment Schemes (EQUAS). The participating laboratories were invited to participate in each exercise by letter and, after acceptance, they received the control materials $(300 \mathrm{mg}$ ), one of low concentration $\left(C_{\text {low }}\right)$ and one of high concentration $\left(C_{\text {high }}\right)$, for each exercise. A total of eight independent samples were sent.

Six weeks after shipment of the samples the laboratories had to submit their results (single and binding values) together with information regarding the analytical method employed and quality controls applied.

Data analysis was performed using the SPSS Statistics software (IBM Corporation, version 19). Values outside 1.5 box lengths from the lower or upper hinge of the box (H-spread, interquartile range of all reported values) were considered to be outliers and were excluded from the evaluation. The Relative Standard Deviation (RSD) was calculated as a measure of the inter-laboratory variability.

The aim of inter-laboratory comparison investigations is to measure the comparability (the degree of variation in analytical results) of participating laboratories. The consensus value, calculated as the mean of the results of the participants after exclusion of the outliers, was used as reference. Results from the ICIs were regarded as valid if they were within the range of the twofold standard deviation of the consensus value for both concentration levels in each round.

External quality-assessment schemes are used to improve the accuracy (the ability to quantify the actual analyte concentration in the sample (true value) of analytical results. Reference Laboratories (RLs) were used to derive assigned values as approximations of the true values. In EQUAS, the accuracy was evaluated by comparing results with the assigned values (calculated from the results of the reference laboratories). The RLs were laboratories with a worldwide reputation and proven excellence through peerreviewed publications and/or experience in organization of wellknown international inter-laboratory exercises (Table 1 ).

The criterion for a successful performance in the EQUAS was that results should be within the threefold standard deviation of the assigned value for each concentration level.

\subsubsection{Overall qualification criteria}

The COPHES/DEMOCOPHES coordination team and the QAU agreed the criteria for defining qualified laboratories (those that could analyze the DEMOCOPHES samples), namely successfully completing at least either one ICI and one EQUAS or two EQUAS.

\subsubsection{Results communication and discussion}

Laboratories received the results by e-mail in a letter containing the individual results, consensus/assigned values and tolerance ranges. After each exercise, a web conference based on the program Elluminate Live (Blackboard Inc., Washington, DC, USA) was held in order to promote communication between the participants and the QAU.

\subsection{Training}

Following the aim of COPHES, namely "to develop a coherent approach on HBM in Europe", and to support capacity building, it was agreed from the outset that each biomarker should be 
Table 1

Reference laboratories involved in the COPHES/DEMOCOPHES EQUAS.

\begin{tabular}{|c|c|}
\hline Reference laboratory & Location \\
\hline Alaska State Public Health Laboratory & Anchorage, USA \\
\hline First Nations and Inuit Health Branch Laboratory (FNIHB) & Ontario, Canada \\
\hline National Institute for Minamata Disease (NIMD) & Kumamoto, Japan \\
\hline RTI International & Triangle, USA \\
\hline School of Medicine and Dentistry - University of Rochester & Rochester, USA \\
\hline $\begin{array}{l}\text { Center de Toxicologie du Québec (CTQ) of the Institut Na- } \\
\text { tional de Santé Publique du Québec (INSPQ) }\end{array}$ & Quebec, Canada \\
\hline
\end{tabular}

analyzed, if feasible, in laboratories within the border of each participating country. At the very beginning of the study, the QAU prepared an inventory of potential laboratories and their capacities, which showed high disparities among them. As a result, special efforts were dedicated to capacity building and the training of less-experienced laboratories.

Representatives of the national studies (responsible for national fieldworker team training) attended a training course on fieldwork. Attendees were trained in general precautions to be considered during the pre-analytical phase by conducting a practical exercise. Special attention was paid to critical aspects, such as the region of the scalp to sample, how to immobilize the lock without invalidating the sample (maintaining the portion to be analyzed free) and what amount of sample should be collected (http://www.eu-hbm.info/cophes/project-work-packages/train ings-agendas-and-presentations).

In addition, each of the four quality-assurance exercises was accompanied by a web conference. Web conferences were the main training tool for the analytical phase since they allowed lessexperienced laboratories to discuss and exchange experiences with other laboratories and the QAU in order to improve their analytical method.

The QAU helpdesk offered technical support for pre-analytical and analytical questions throughout the project.

\section{Results and discussion}

The main goal of the COPHES/DEMOCOPHES projects is to demonstrate that a harmonized approach to HBM can be achieved in Europe. One of the biomarkers within the scope of the pilot study was mercury in hair since mercury contamination is a public health concern due to its toxicity and widespread exposure to methylmercury through the diet. In addition, the target population for DEMOCOPHES (woman of childbearing age and children) is the group most vulnerable to the effects of methylmercury.

As the pilot study was designed as a multicentre study, it was necessary to define the procedures to be applied in the 17 participating countries to prevent a lack of harmonization in sampling and chemical analysis influencing the final results and conclusions derived from them. In addition, the concentration of mercury in hair varies with dietary habits, which are quite different as regards fish consumption (Castaño et al., in this issue), therefore a wide range of concentrations was expected in the DEMOCOPHES target population. Depending on the frequency of consumption and fish species, levels can vary from $<0.5 \mu \mathrm{g} / \mathrm{g}$ for non-fish eaters to $1-$ $2 \mu \mathrm{g} / \mathrm{g}$ for those with low to moderate consumption. The concentration can exceed $10 \mu \mathrm{g} / \mathrm{g}$ for high fish consumers (UNEP/ WHO, 2008). This scenario made it necessary for strict control measures to be put into place during both the analytical and preanalytical phase, therefore different tools were developed to harmonize and control the latter.

The SOP for hair sampling was crucial to ensure a common method in all fieldwork teams and to control specific methodological issues. For example, as the scalp hair growth rate varies depending on the region of the scalp (Harkey, 1993), it was essential to define the area for sampling. Another important aspect was immobilization of the lock since the analysts had to know which end was the one closest to the scalp. Furthermore, the segment of the sample to be analyzed, previously defined in the study protocol as the first $3 \mathrm{~cm}$, had to be free of the adhesive tape used to immobilise the lock otherwise the sample would be invalidated. Previous studies had shown that the way in which the adhesive tape is attached is frequently a tricky issue in sampling and the training sessions gave the opportunity to emphasize this and practice the sampling technique. The amount of sample was probably the most problematic issue. A minimum amount of $200 \mathrm{mg}$ of hair was strongly recommended in the pre-analytical SOP for mercury as a lower amount of hair may increase the LOQ. Generally, the fieldworkers do not analyze the samples and thus they are not aware of the importance of the amount of hair necessary to perform the analysis. When using other biological matrices, urine for example, it's easier to indicate the minimum amount to collect as the vessel can be marked with a line indicating the required volume and samplers can verify if the minimum amount has been collected. However, this approach cannot be applied in the case of hair. Despite the QAU recommendation and the training sessions, one DEMOCOPHES country collected too little hair in most of their samples and, as a result, a high percentage of their results were below the LOQ value.

With regard to the controversy concerning internal and external mercury and sample washing, during the discussion of the study protocol it was agreed to avoid washing hair. This decision was taken to simplify the procedure and also because there is not yet a consensus washing method and some procedures have shown the possibility that endogenous mercury present in hair may be removed (Veiga and Baker, 2004; Li et al., 2008; Poulin and Gibbs, 2008). Similarly, it was specifically established in the project protocol that selected sampling locations (urban and rural) near industrial hot-spots should be excluded (Becker et al., 2014). Despite that, as an additional control, a hair-sampling questionnaire was used and information about the last wash prior to sampling and other data, such as natural hair color and structure, use of dyes or any problem encountered during sampling, was recorded for each sample. None of the variables included in the hair questionnaire were found to have an effect on mercury levels after data analysis (unpublished data).

The preferred technique for mercury analysis in the participating laboratories was Atomic Absorption Spectrometry (AAS), mainly coupled with thermal decomposition and, to a lesser extent, Cold Vapor generation (CV-AAS). More than the $50 \%$ of the laboratories used Direct Mercury Analyzers (DMA) and only three used Inductively Coupled Plasma Mass Spectrometry (ICP-MS). Although ICP-MS is more sensitive than AAS, the LOQ achievable by direct mercury analysis is low enough to analyze mercury concentrations present in the target population and has the advantage of not requiring sample pre-treatment or extraction. In addition, the short analysis times allow high sample throughput. The limit of quantification among participating laboratories ranged from 0.00003 up to $0.1 \mu \mathrm{g} / \mathrm{g}$.

From an analytical point of view, the most straightforward strategy for obtaining comparable results is to analyze all samples in one well-experienced laboratory instead of the multi-laboratory approach. However, this approach would have been contrary to the aim of COPHES and the goal established in the European Environment \& Health Action Plan. In addition, there would have been ethical concerns and economic drawbacks in sending human samples outside national borders. The multi-center approach defined made it necessary to check the state-of-the-art in all 
participating countries. Although some laboratories would have been excluded solely on the basis of the data from the inventory, the QAU emphasized the need for capacity building by supporting less-experienced laboratories, thereby allowing them to acquire the skills required to comply with established quality criteria in hair analysis.

Analytical SOPs are key tools for improving the comparability and reliability of analytical results and constituted a basic measure in the harmonization process. However, as a SOP is not sufficient to ensure the comparability of data, additional control measures were applied. Thus, every laboratory was required to participate in the external quality-assessment exercises, which were actually the key tool to ensure the comparability of results.

The two ICIs and two EQUAS were implemented in less than a year and a total of 18 laboratories and seven reference laboratories took part. More than 150 samples of native, non-spiked hair control materials with an RSD of less than 3\% were carefully prepared.

Contrary to what was observed for some of the biomarkers measured in urine (Schindler et al., 2014), no significant drawbacks were encountered during analysis of total mercury in hair during the external quality-assessment exercises (Table 2). One laboratory had to withdraw after the second ICI and all laboratories except one qualified according to the established criteria (94\% success rate). The relative standard deviations of less than $14 \%$ reflect a good comparability of the data for the relevant concentration range. Since a high percentage of the participating laboratories completed the exercise successfully, only a slight difference in comparability was found upon selection of the qualified laboratories.

Evaluation of the EQUAS results was supported by the data provided by highly experienced and world-renowned Reference Laboratories (RLS), which also performed the analysis using different methodologies, namely DMA, CV-AAS and Cold Vapor Atomic Fluorescence Spectroscopy (CV-AFS). The results from these RLs showed relative standard deviations of $7.18-17.03 \%$, which were somewhat higher than those obtained by the participating laboratories (4.04-13.55\%). Qualified laboratories showed RSDs as good as, or even better than, those obtained by RLs.

The same control material was used for some rounds. The repeated samples served as an extra control to test the intra- a

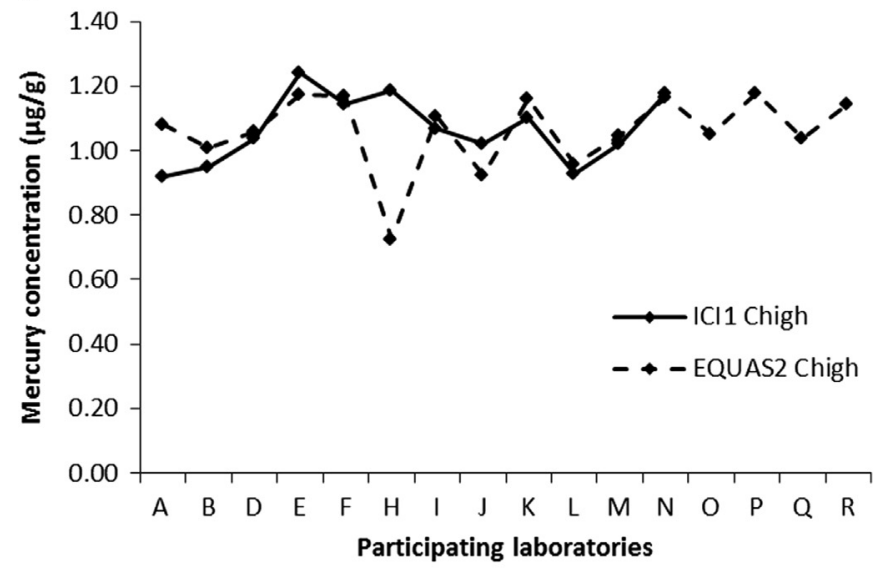

b

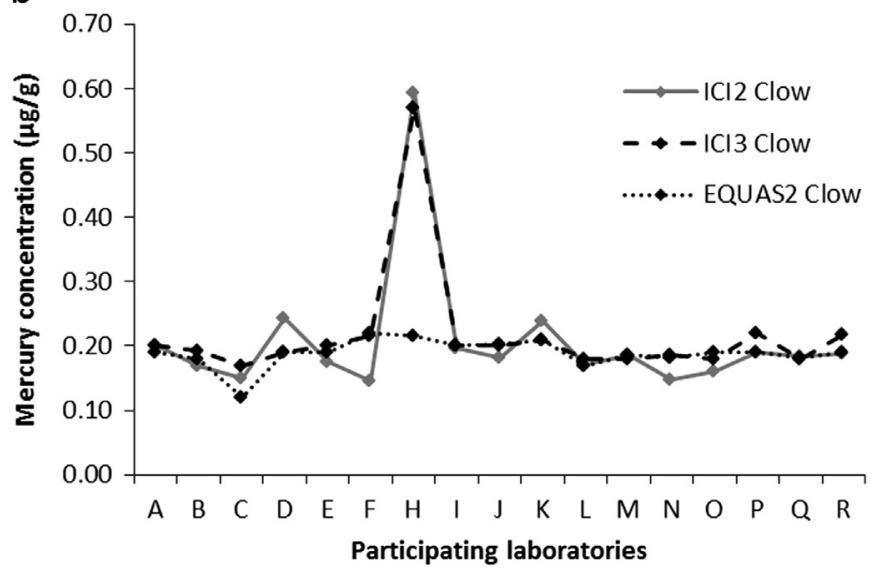

Fig. 1. Results from the intra-variability control test. (a) Repeated sample with low concentration. (b) Repeated sample with high concentration (Laboratories $O, P, Q$ and $R$ did not participate in ICI 1 ).

laboratory variability (Fig. 1). The RSD for the values reported by each participant in the intra-laboratory control were in the ranges $1.04-46.17 \%\left(C_{\text {low }}\right)$ and $0.73-34.33 \%\left(C_{\text {high }}\right)$ (up to $21.45 \%$ and $11.31 \%$, respectively, when removing the outlier value). The

Table 2

Results from the external quality-assessment exercises for mercury in hair

\begin{tabular}{|c|c|c|c|c|c|c|c|c|}
\hline & \multicolumn{4}{|c|}{$C_{\text {low }}(\mu \mathrm{g} / \mathrm{g})$} & \multicolumn{4}{|c|}{$C_{\text {high }}(\mu \mathrm{g} / \mathrm{g})$} \\
\hline & ICI 1 & ICI 2 & EQUAS 1 & EQUAS 2 & ICI 1 & ICI 2 & EQUAS 1 & EQUAS 2 \\
\hline Consensus value & 0.74 & 0.18 & 0.20 & 0.19 & 1.05 & 1.47 & 0.70 & 1.08 \\
\hline Assigned value & & & 0.20 & 0.18 & & & 0.76 & 1.08 \\
\hline \multicolumn{9}{|l|}{ Participating labs } \\
\hline $\boldsymbol{n}_{\text {participating labs }}$ & 12 & 18 & 17 & 17 & 12 & 17 & 17 & 17 \\
\hline $\boldsymbol{n}_{\text {participating labs, outliers excluded }}$ & 12 & 15 & 16 & 17 & 12 & 13 & 12 & 16 \\
\hline \% $\mathbf{R S D}_{\text {participating labs }}$ & 8.90 & 13.55 & 7.87 & 11.64 & 11.31 & 6.61 & 4.04 & 7.30 \\
\hline \multicolumn{9}{|l|}{ Qualified labs } \\
\hline $\boldsymbol{n}_{\text {qualified labs }}$ & & 16 & 16 & & 16 & 16 & & \\
\hline$\% \mathbf{R S D}_{\text {qualified labs }}$ & & 7.87 & 11.54 & & 4.23 & 7.30 & & \\
\hline \multicolumn{9}{|l|}{ Reference labs } \\
\hline $\boldsymbol{n}_{\text {reference labs }}$ & & 6 & 6 & & 6 & 6 & & \\
\hline \% $\mathbf{R S D}_{\text {reference labs }}$ & & 7.45 & 17.03 & & 7.18 & 8.76 & & \\
\hline
\end{tabular}

RSD: relative standard deviation; $n$ : number; $C_{\text {low }}$ : low concentration; $C_{\text {high }}$ : high concentration

Note: different control samples were used as $C_{\text {low }}$ and $C_{\text {high }}$ in each exercise 
laboratory with the highest RSD for both concentration levels was the only one that did not qualify for analyzing DEMOCOPHES samples according to the defined criteria.

Although participation in ICIs and EQUAS is a common practice for analytical laboratories, a quality-assurance program that covers the full project from sampling to results communication was pioneering within Europe. In the framework of COPHES/DEMOCOPHES, the aim of which is to obtain comparable and reliable results, it seems clear that this kind of quality control is necessary and crucial to the success of the project as the laboratories involved had to prove their proficiency before analyzing real samples. Despite this, the framework for implementing the ICI/EQUAS and, more importantly, time constraints required a tailor-made design with significant differences as regards commercial qualityassurance schemes, for example support via web conferences, the training and the SOPs, or the use of native (non-spiked) control material reflecting population levels.

Web conferences, which are used to connect many participants in different locations in real time, were a completely new mode of operation in external quality-assessment exercises. From a practical point of view, they proved to be highly efficient in terms of time and cost while maintaining the advantages of face-to-face meetings, and they supported open communication within a multicultural and multinational network. Exchange of knowledge and experience was facilitated in a natural manner, thus proving particularly valuable with respect to capacity building. It further turned out that such web conferences (depending on how they are carried out) can even guarantee the anonymity of the participants. Anonymity is a decisive criterion for successful quality assurance, because only anonymity guarantees objectivity of results without exposing laboratories that produced unreliable results.

The experience gained in DEMOCOPHES is of particular current interest, especially as regards implementation of the Minamata Convention. As can be seen, hair is an easy matrix to collect, store and even transport. In addition, total mercury in hair analysis is a relatively simple technique that does not require highly sophisticated equipment and, has been shown with the tools used, even less-experienced laboratories can acquire the skills required to obtain comparable results. These aspects are particularly important in order to actively involve countries with a lower degree of technological development in implementation of the treaty and in protection of their population.

This will be the major achievement to get everyone on board and moving in the same direction.

\section{Conclusions}

The activities concerning harmonizing mercury exposure assessment in hair as part of the COPHES/DEMOCOPHES projects have demonstrated that a coherent approach to human biomonitoring in Europe is feasible. Indeed, the results have shown that harmonization of mercury analysis in hair does not present significant difficulties, with a lab success rate of $94 \%$.

The fact that minor problems were encountered in the preanalytical phase highlights the need for standard operating procedures and external quality-assessment exercises even when using a well-established biomarker such as mercury.

The approach and procedures elaborated and tested in COPHES/ DEMOCOPHES could be used as a blueprint for future multicentre HBM studies involving the analysis of hair as a biological matrix.

Mercury in hair is a strongly recommended biomarker for assessing mercury exposure within a worldwide framework, such as implementation of the Minamata Convention.

\section{Funding}

This work would not have been possible without the co-funding from the Spanish Ministry of Agriculture, Food and the Environment (MAGRAMA) and The Institute of Health Carlos III (ISCIII) Agreement, SEG 1251/07 and SEG 1112/10. The COPHES project that provided the operational and scientific framework was funded by the European Community's Seventh Framework Program - DG Research (Grant Agreement Number 244237 - www. eu-hbm.info). The DEMOCOPHES project (LIFE09 ENV/BE/000410) is jointly funded by the European Commission program LIFE+ (50\%), with the remaining $50 \%$ being provided by the participating countries (see the national implementation websites accessible via http://www.eu-hbm.info/democophes/project-partners).

\section{Acknowledgements}

The COPHES QAU members would like to thank all DEMOCOPHES laboratories for their cooperation and especially the Reference Laboratories that kindly participated in EQUAS exercises without receiving any payment and despite not being partners in the COPHES projects.

\section{References}

ATSDR, 2001. Hair Analysis Panel Discussion: Exploring the State of the Science Summary Report, Atlanta, GA, Agency for Toxic Substances and Disease Registry.

Becker, K., Seiwert, M., Casteleyn, L., Joas, R., Joas, A., Biot, P., Aerts, D., et al., 2014. A systematic approach for designing a HBM Pilot Study for Europe. Int. J. Hyg. Environ. Health 217, 312-322.

Berglund, M., Lind, B., Björnberg, K.A., Palm, B., Einarsson, Ö, Vahter, M., 2005. Interindividual variations of human mercury exposure biomarkers: a cross-sectional assessment. Environ. Health 3, 4-20.

Castaño, A., Cutanda, F., Esteban, M., Pärt, P., Navarro, C., Gómez, S., Rosado, M., López, E., López, A., Exley, K., Schindler, B., Govarts, E., Casteleyn, L., KolossaGehring, M., Fiddicke, U., Koch, H.M., Angerer, J., Schoeters, G., Den Hond, E. Sepai, O., Knudsen, L.E., Horvat, M., Aerts, D., Biot, P., Bloemen, L., Joas, A., Joas, R., Diaz, G., Jiménez-Guerrero, J.A., Pirard, C., Charlier, C., Katsonouri, A., Hadjipanayis, A., Cerna, M., Krsková, A., Schwedler, G., Wilhelm, M., Nielsen, J.K. Jensen, J.F., Rudnai, P., Középesy, S., Cullen, E., Davidson, F., Gutleb, A.C., Fischer M.E., Ligocka, D., Janasik, B., Reis, F.M., Lupsa, I.O., Berglund, M., Namorado, S., Gurzau, A.E., Halzlova, K., Jajcaj, M., Tratnik, J.S., Mazej, D., Larsson, K., Lehmann, A., Crettaz, P., Lavranos, G., Posada, M., 2014. Fish consumption patterns and hair mercury levels in children and their mothers in 17 EU countries. Environ. Res. (in this issue).

Clarkson, T.M., Magos, L., 2006. The toxicology of mercury and its chemical compounds. Crit. Rev. Toxicol. 36, 609-662.

COPHES, 〈www.eu-hbm.info/cophes $\rangle$. Consortium to Perform Human Biomonitoring on a European Scale.

Dakeishi, M., Nakai, K., Sakamoto, M., Iwata, T., Suzuki, K., Liu, X.J., Ohno, T., Kurosawa, T., Satoh, H., Murata, K., 2005. Effects of hair treatment on hair mercury - the best biomarker of methylmercury exposure? Environ. Health Prev. Med. 10, 208-212.

DEMOCOPHES, 〈http://www.eu-hbm.info/democophes $\rangle$. Demonstration of a Study to Coordinate and Perform Human Biomonitoring on a European Scale.

EFSA, 2012. Scientific opinion on the risk for public health related to the presence of mercury and methylmercury in food. EFSA J. 10, 2985

EHAP, 2004. The European Environment \& Health Action Plan 2004-2010. COM 416. Available in http://ec.europa.eu/governance/impact/ia_carried_out/docs/ ia_2004/com_2004_0416_1_en.pdf.

ESBIO, 2009. Development of a Coherent Approach to Human Biomonitoring in Europe, Coordination Action, Final Activity Report, Expert Team to Support Biomonitoring in Europe.

Harkey, M.R., 1993. Anatomy and physiology of hair. Forensic Sci. Int. 63, 9-18.

Harkins, D., Susten, A.S., 2003. Hair analysis: exploring the state of the science. Environ. Health Perspect. 111, 576-578.

Horvat, M., Tratnik, J.S., Miklavčič, A., 2012. Mercury: biomarkers of exposure and human biomonitoring. In: Knudsen, Merlo (Eds.), Biomarkers and Human Biomonitoring, Volume I: Ongoing Programs and Exposures. RSC, Cambridge, UK.

Joas, R., Casteleyn, L., Biot, P., Kolossa-Gehring, M., Castano, A., Angerer, J., Schoeters, Sepai, O., Knudsen, L.E., Joas, A., Horvat, M., Bloemen, L., 2012. Harmonised human biomonitoring in Europe: Activities towards an EU HBM framework. Int J. Hyg. Environ. Health 215, 172-175. 
Li, Y.F., Chen, C., Li, B., Wang, J., Gao, Y., Zhao, Y., Chai, Z., 2008. Scalp hair as a biomarker in environmental and occupational mercury exposed populations: suitable or not? Environ. Res. 107, 39-44.

McDowell, M.A., Dillon, C.F., Osterloch, J., Bolger, P.M., Pellizzari, E., Fernando, R., Montes de Oca, R., Schober, S.E., Sinks, T., Jones, R.L., Mahaffey, K.R., 2004. Environ. Health Perspect. 112, 1165-1171.

Poulin, J., Gibbs, H., 2008. In: Prüss-Üstün, A. (Ed.), Assessing the Environmental Burden of Disease at National and Local Levels. World Health Organization, Geneva.

Schindler, B.K., Esteban, M., Koch, H.M., Castaño, A., Koslitz, S., Cañas, A., Casteleyn, L., Kolossa-Gehring, M., Schwedler, G., Schoeters, G., Hond, E.D., Sepai, O., Exley, K., Bloemen, L., Horvat, M., Knudsen, L.E., Joas, A., Joas, R., Biot, P., Aerts, D. Lopez, A., Huetos, O., Katsonouri, A., Maurer-Chronakis, K., Kasparova, L., Vrbík, K., Rudnai, P., Naray, M., Guignard, C., Fischer, M.E., Ligocka, D., Janasik, B., Reis, M.F., Namorado, S., Pop, C., Dumitrascu, I., Halzlova, K., Fabianova, E., Mazej, D. Tratnik, J.S., Berglund, M., Jönsson, B., Lehmann, A., Crettaz, P., Frederiksen, H. Nielsen, F., McGrath, H., Nesbitt, I., De Cremer, K., Vanermen, G., Koppen, G., Wilhelm, M., Becker, K., Angerer, J., 2014. The European COPHES/DEMOCOPHES project: towards transnational comparability and reliability of human biomonitoring results. Int. J. Hyg. Environ. Health 217, 653-661.

UBA, 2005. Hair analysis in environmental medicine. German Federal Environmental Agency. Opinion of the human biomonitoring Commission of the German Federal Environment Agency. Bundesgesundheitsbl-Gesundheitsforsch -Gesundheitsschutz 48, 246-250. http://dx.doi.org/10.1007/s00103-0040984-3.

UNEP/WHO, 2002. Global Mercury Assessment. United Nations Environment Program/World Health Organization, Geneneva.

UNEP/WHO, 2008. Guidance for Identifying Populations at Risk from Mercury Exposure. United Nations Environment Program/World Health Organization, Geneva.

Veiga, M.M., Baker, R.F., 2004. Global Mercury Project. Protocols for Environmental and Health Assessment of Mercury Released by Artisanal and Small-scale Gold Miners. United Nations Industrial Development Organization, Vienna, Austria. 